\title{
Intra Oral Approach for Complex \& Compound Odontomas (Large or Small)
}

\author{
Parveen Akhter Lone*, Indumeet Kour, Annuradha Gandral \\ Department of Oral \& Maxillofacial Surgery, Indira Gandhi Government Dental College Amphalla, Affiliated to \\ University of Jammu, Jammu, India \\ Email: ${ }^{*}$ Parveenlone@gmail.com, ${ }^{*}$ Parveen.lone@yahoo.com
}

Received 23 May 2014; revised 18 June 2014; accepted 15 July 2014

Copyright $@ 2014$ by authors and Scientific Research Publishing Inc.

This work is licensed under the Creative Commons Attribution International License (CC BY).

http://creativecommons.org/licenses/by/4.0/

(c) ()

\begin{abstract}
Odontoma by definition alone refers to any tumour of odontogenic origion. These are considered to be hamartomatous malformation rather than true neoplasm and are generally asymptomatic. World Health Organization defines a lesion as the complex odontoma: when the calcified dental tissues are simply arranged in an irregular mass bearing no morphologic similarity to rudimentary teeth. Compound odontomas: composed of all odontogenic tissues in an orderly pattern, which result in many teeth-like structures, but without morphologic resemblance to normal teeth. The goal of this article is to report two cases of the complex odontomas erupted in oral cavity and compound odotome with impacted teeth. The article also aims to report that intra oral approach for removal of odontomas complex or compound small or large is better than extra oral approach.
\end{abstract}

\section{Keywords}

Intra Oral, Hamartomas, Odontogenic, Odontomas

\section{Introduction}

The term odontoma was coined by Paul Brocha in 1867. By definition odontomas are hamartomas of odotogenic origion and are usually considered as developmental anomalies [1]. Complex odontomas tend to occur more commonly in anterior maxilla [2]. An odontoma is a growth in which both epithelial cells exhibit complete differentiation with the result that functional ameloblast and odontoblast form enamel and dentin [3]. Odontomas are benign tumours of odontogenic origin consisting of enamel, dentin, cementum and pulpal tissue, and constitute for $22 \%$ of all odontogenic tumors [4]. They are characterized by their slow growth and non aggressive nature. During the development of the tumor, enamel and dentin can be deposited in such a way that the resulting

${ }^{*}$ Corresponding author. 
structures show an anatomic similarity to normal teeth, in which case the lesion is classified as a compound odontoma. However, when the dental tissues form a simple irregular mass occurring in a disorderly pattern, it is described as a complex odontoma [5]. Compound odontomas appear more frequently than complex odontomas [6] [7]. Compound odontomas [8] consist of tooth-like structures that radiographically appear as opacities. Complex odontomas comprise a mixture of odontogenic tissues without dental organization [9]. Clinically, three types of odontomas are recognized in the literature: central (intraosseous) odontoma, peripheral (extraosseous or soft tissue) odontoma, and erupted odontoma [10]. Odontomas are often associated with an unerupted or impacted tooth and the failure of a permanent tooth to erupt is the most common clinical manifestation [11]. Odontomas generally appear as small, solitary or multiple radiopaque lesions detected during routine radiographic examination that may cause disturbances in tooth eruption, such as impaction, delayed eruption or retention of primary teeth [12]. Although it is rare, there may be spontaneous eruption of an odontoma into the oral cavity which leads to pain and inflammation of adjacent soft tissues and can be confused with some bony lesion [13].

\section{Case Reports}

\subsection{Case 1}

A 15-year-old male patient reported with a complaint of facial deformity \& pain in the left lower back tooth region since 3 months. Pain was spontaneous in onset, dull, intermittent, non-radiating, aggravates on chewing and relieved by itself after few minutes. His past dental and medical history was not significant. Extra oral examination revealed facial deformity on left side of lower face, non-tender, bony hard, with smooth surface \& regular borders. Swelling was extending from left body of mandible to ramus region, \& excluding lower border of mandible. It was $3 \mathrm{~cm}$ in size. Intraoral examination revealed an irregularly shaped yellowish brown mass, approximately $3 \mathrm{~cm}$ in maximum dimension over the left molar area. 2nd premolar \& all the permanent molars were absent. The surrounding mucosa was inflamed. On palpation the brown mass was bony hard in consistency and was covered with calculus. Buccal \& lingual cortical plates expanded. Panoramic radiograph revealed a mixed radiopaque-radiolucent area roughly square in shape and approximately $3.5 \mathrm{~cm}$ in maximum diameter in the left mandibular molar area, the internal structure of which is largely radiopaque showing irregular mass of calcified tissue with varying degree of radiolucency. Mandibular first molar \& premolars were impacted \& second \& third molars were missing. Lower border of mandible is very thin \& intact. Complex odontoma was diagnosed, Patient was planned under general anesthesia, intral oral horizontal incision, \& anterior vertical incision was given on alveolar mucosa, odontoma was enucleated along with first molar, primary closure was done \& healing was uneventful (Figures 1-5).

\subsection{Case 2}

A 40 years old male reported with pain \& swelling in right side lower jaw. O/E right first molar was missing, swelling \& tenderness was present was present in RT buccal mucosa. OPG revealed around $2 \mathrm{~cm} \times 1 \mathrm{~cm}$ in size. Complex odontoma was diagnosed \& intra orally odontoma was removed by intra oral approach under local anesthesia (Figures 6-8).

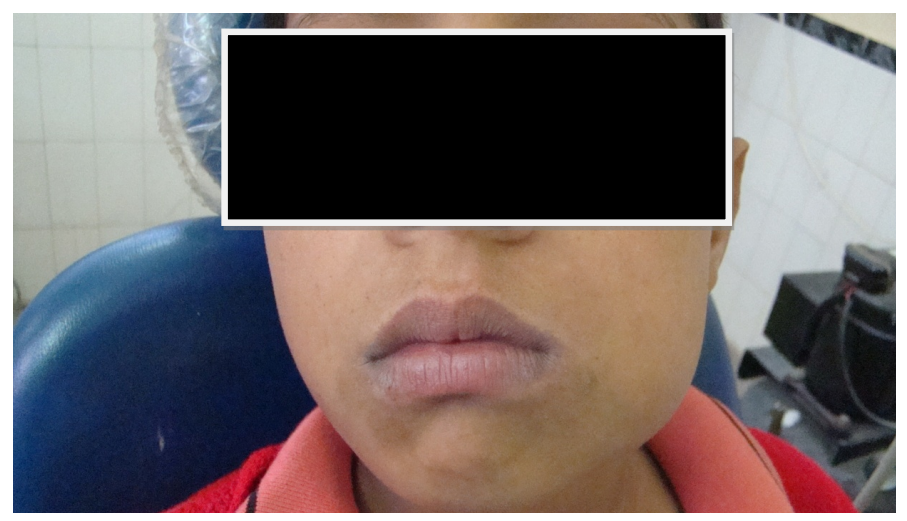

Figure 1. Pre operative photo showing facial assymtery on left side. 


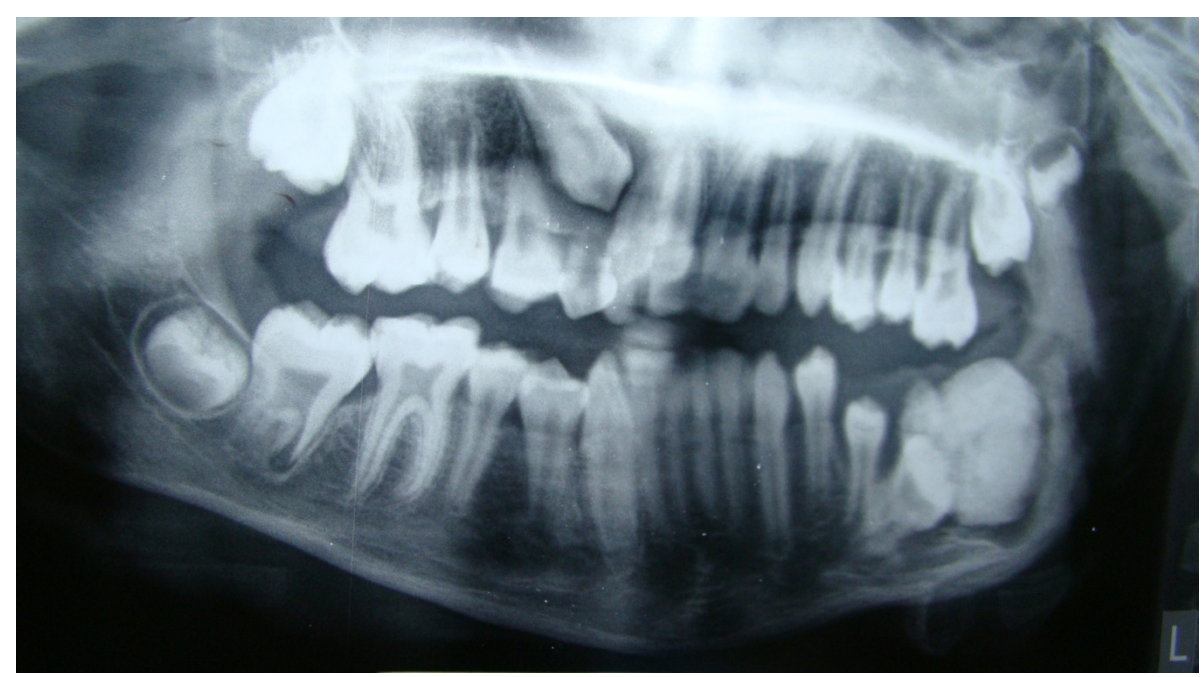

Figure 2. Pre operative radiograph showing odontoma with impacted teeth.

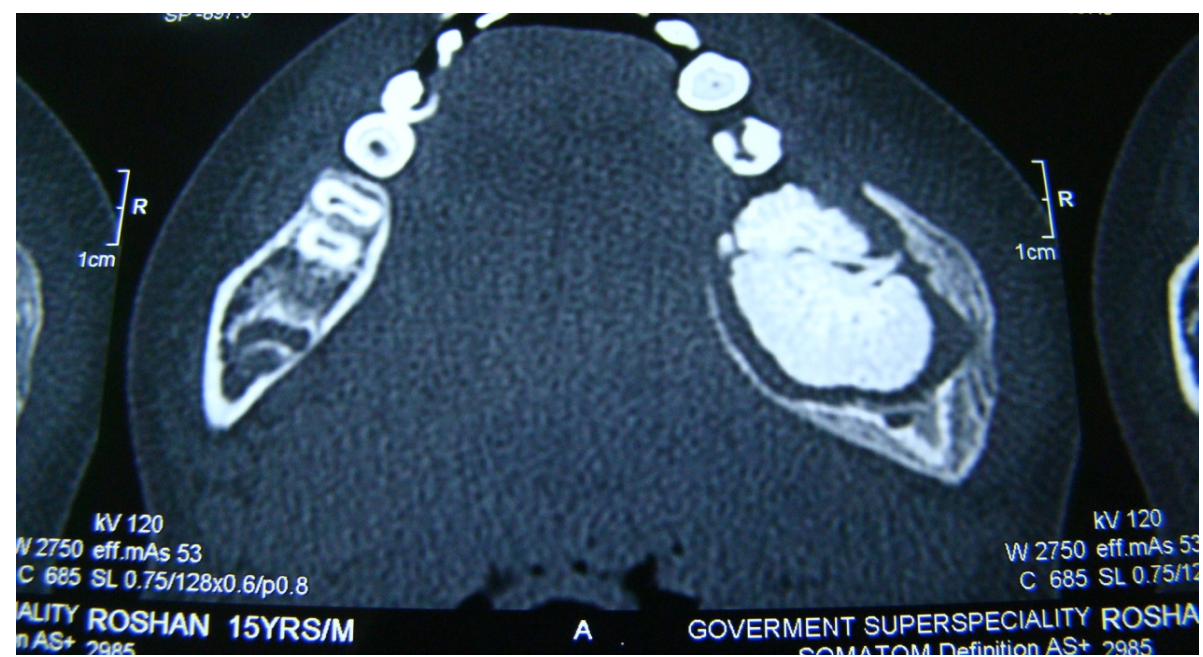

Figure 3. CT scan showing odontoma.

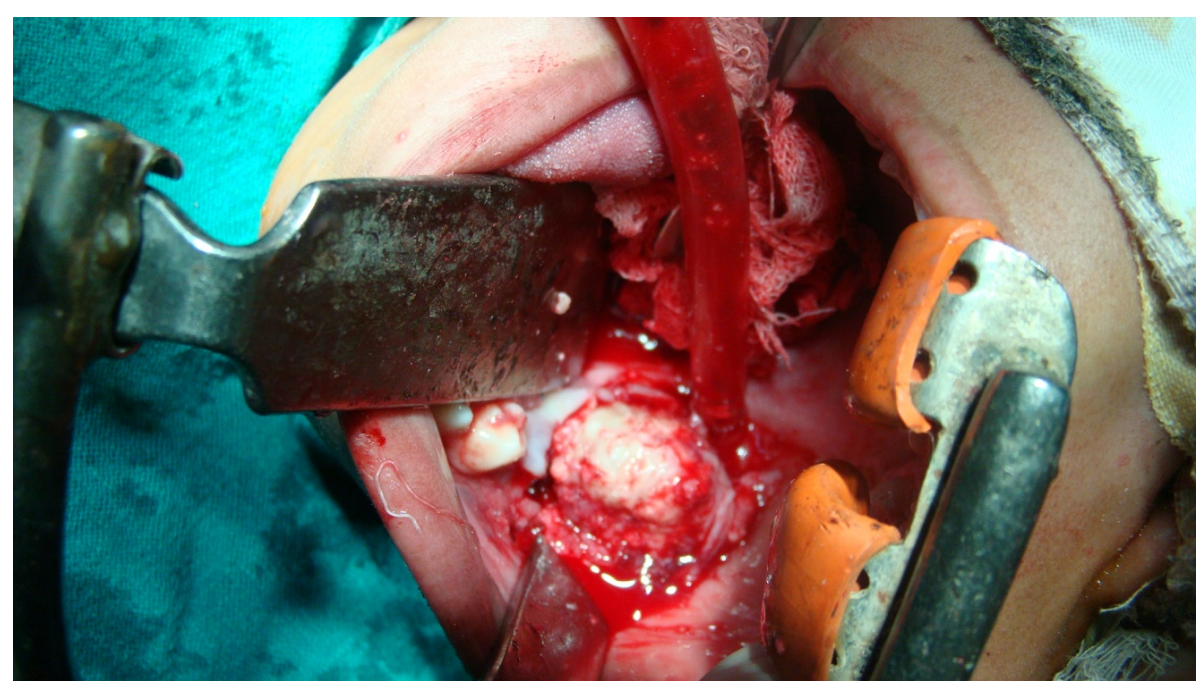

Figure 4. Intra operative photo showing exposed mass. 


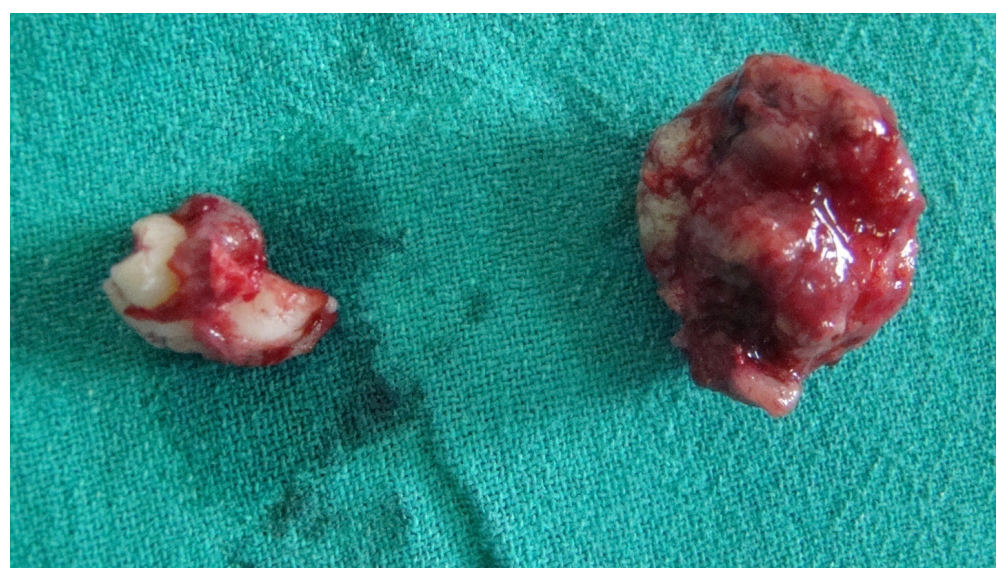

Figure 5. Odontoma \& impacted teeth.

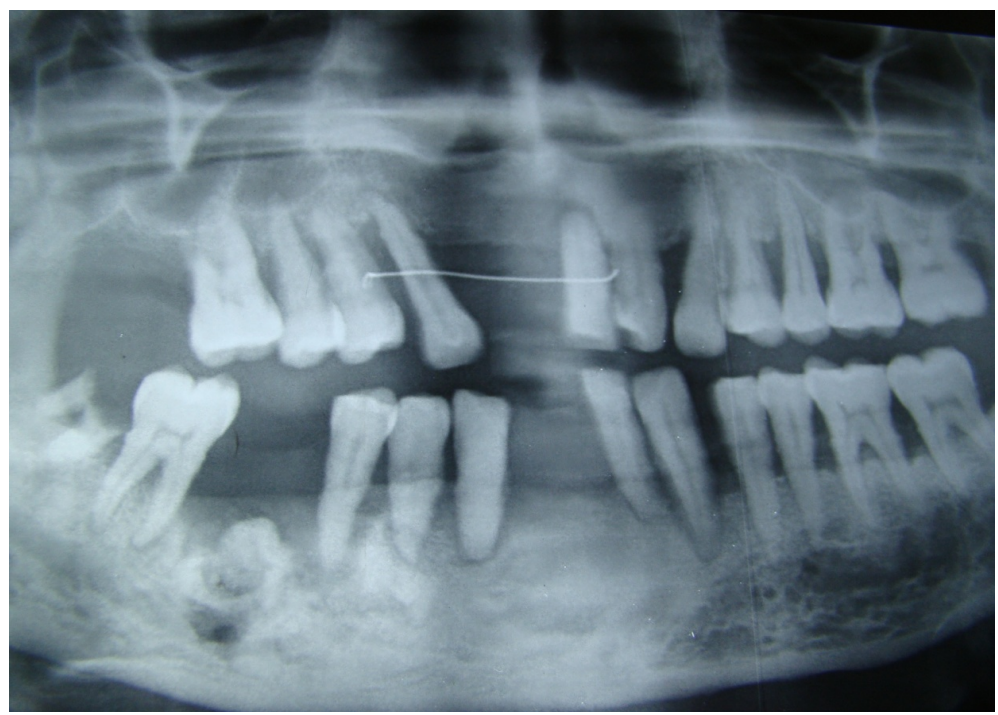

Figure 6. Preoperative radiograph showing small odontoma.

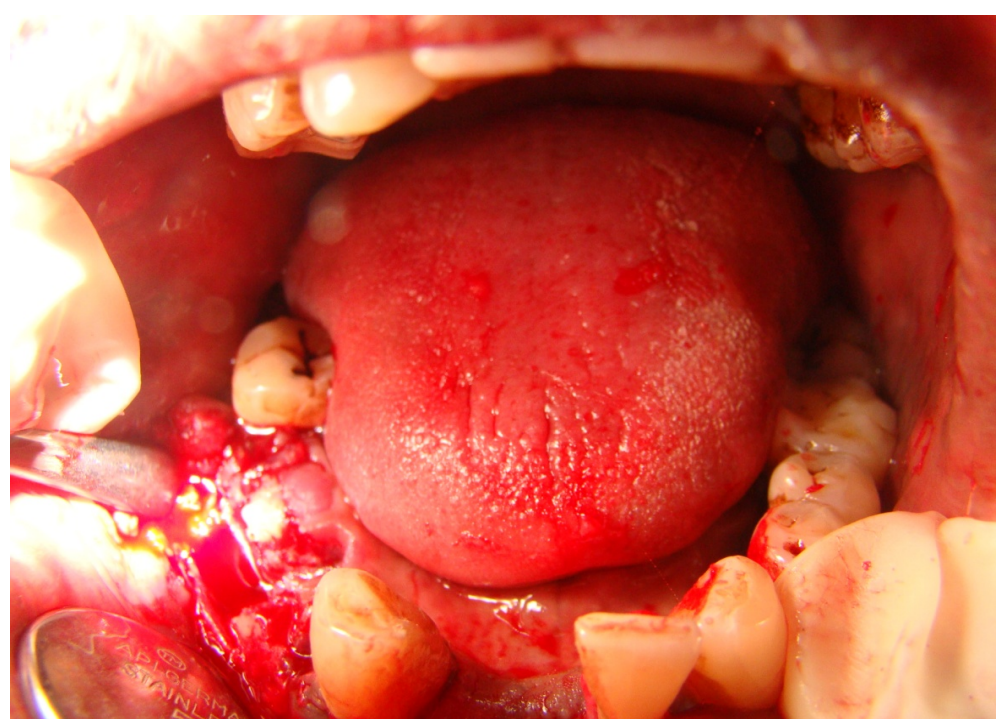

Figure 7. Intraoperative radiograph showing odontoma. 


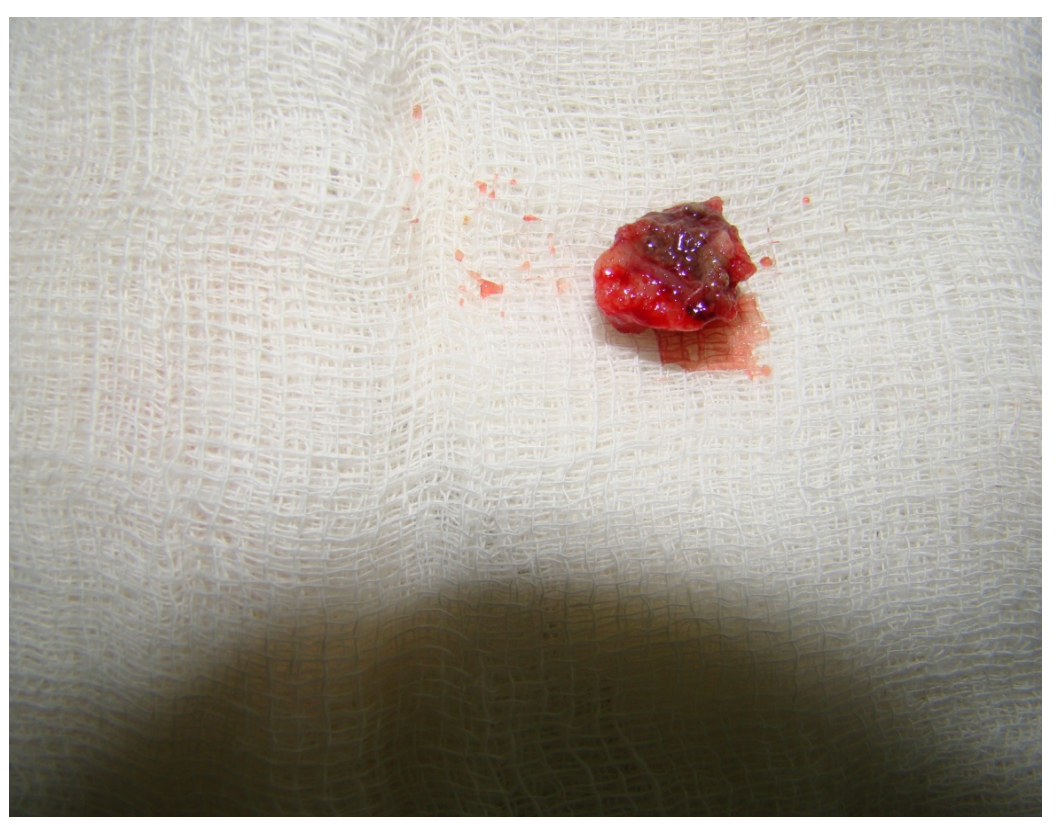

Figure 8. Removed odontoma.

\subsection{Case 3}

An 18-year-old male reported to our department with complaint of missing left maxillary central incisor \& swelling, no history of pain swelling etc. OPG revealed central incisor impacted with a radioopaque structure, impacted tooth along with tooth like structures was removed intra orally under local anesthesia, histopathology confirmed compound odontoma (Figures 9-13).

\subsection{Case 4}

A 24-year-old female reported to the department of oral \& maxillofacial surgery with complaint of missing tooth on right side lower posterior region. O/E small tooth like structure was visible. OPG revealed multiple teeth like structure surrounded by radiolucent border with RT impacted first premolar impacted, compound odontoma was diagnosed. The patient was planned under local anesthesia, intra orally, three sided flap was raised, ten teeth like structure were removed. Impacted first premolar was exposed \& removed. Wound was sutured (Figures 14-16).

\section{Results}

Amongest four cases two males were below twenty years old, one was large erupted complex odontoma around $3 \mathrm{~cm} \times 5 \mathrm{~cm}$ in size with impacted first molar \& second premolar \& missing second \& third molars on radiograph\& other had small $2 \mathrm{~cm} \times 2 \mathrm{~cm}$ compound odontoma.

Two cases were between twenty \& fifty years, one 24 years female had compound \& 45 years male had a complex odontoma.

All were diagnosed on radiographic investigations.

Patients with compound odontoma were assymptomatic where as patients with complex odontomas had pain swelling, both were erupted in oral cavity.

Amongest four patients one was female \& three were males.

Three lesions were in posterior region in mandible, two complex \& one compound, where as one compound odontoma was in maxillary anterior region.

Two odontomas were present on right side (1 complex, 1 compound), one complex large odontoma on left side of mandible, where as one compound odontoma was present in maxillary anterior region.

All odontomas small or large were treated by intra oral approach healing was seen by primary intension \& was uneventful.

Three cases were done under local anesthesia \& one large lesion was removed under general anesthesia. 


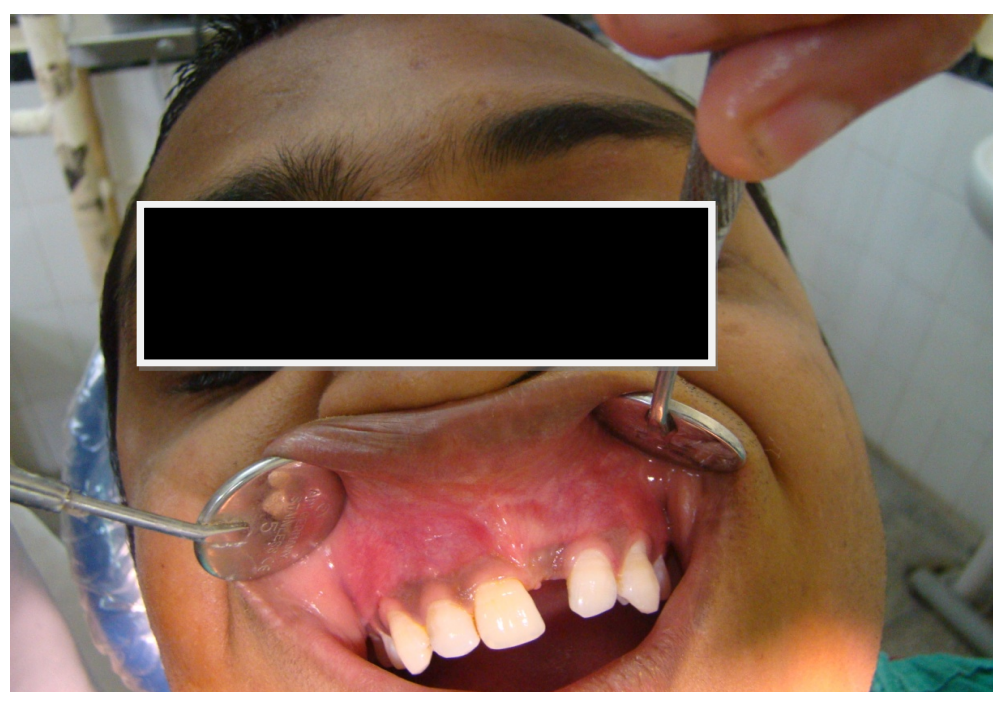

Figure 9. Pre operative photo showing missing central incisor.

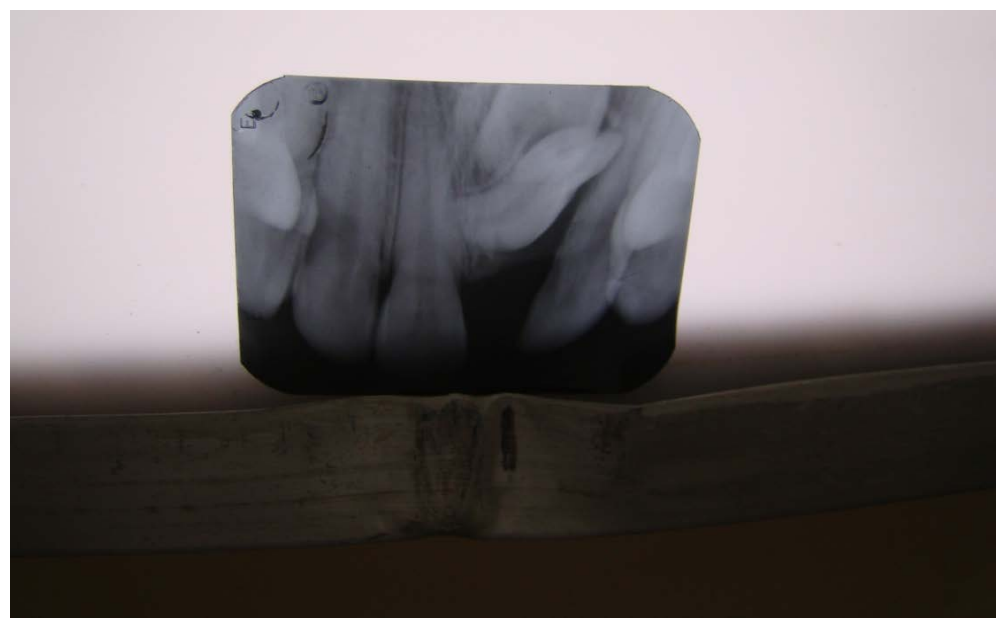

Figure 10. I.O.P.A. showing impacted incisor with odontoma.

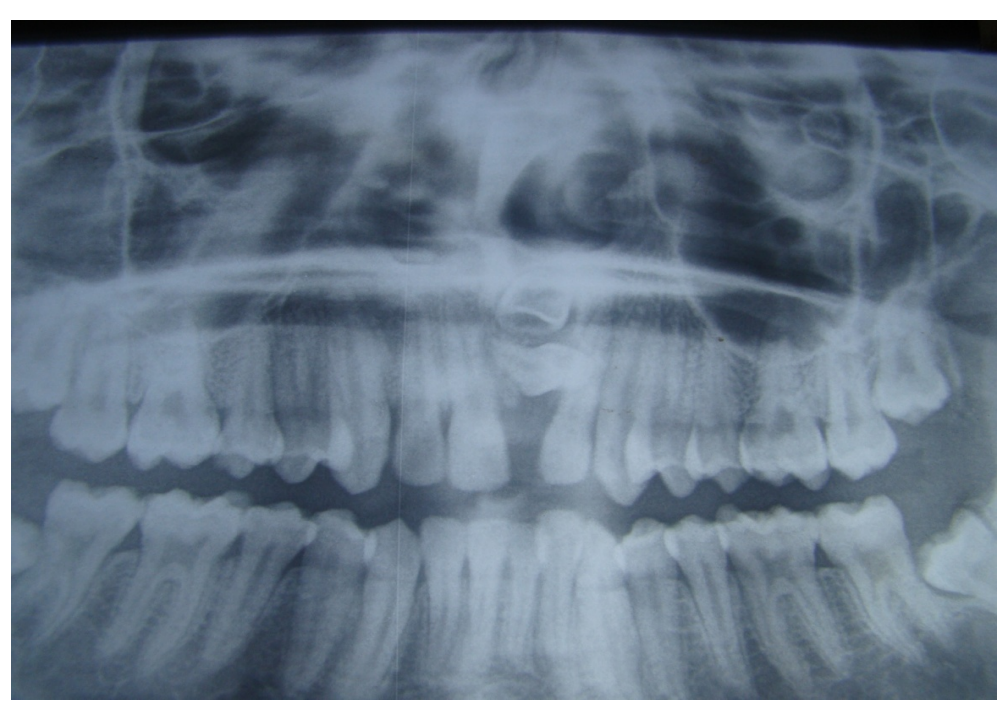

Figure 11. OPG showing impacted central incisor \& odontoma. 


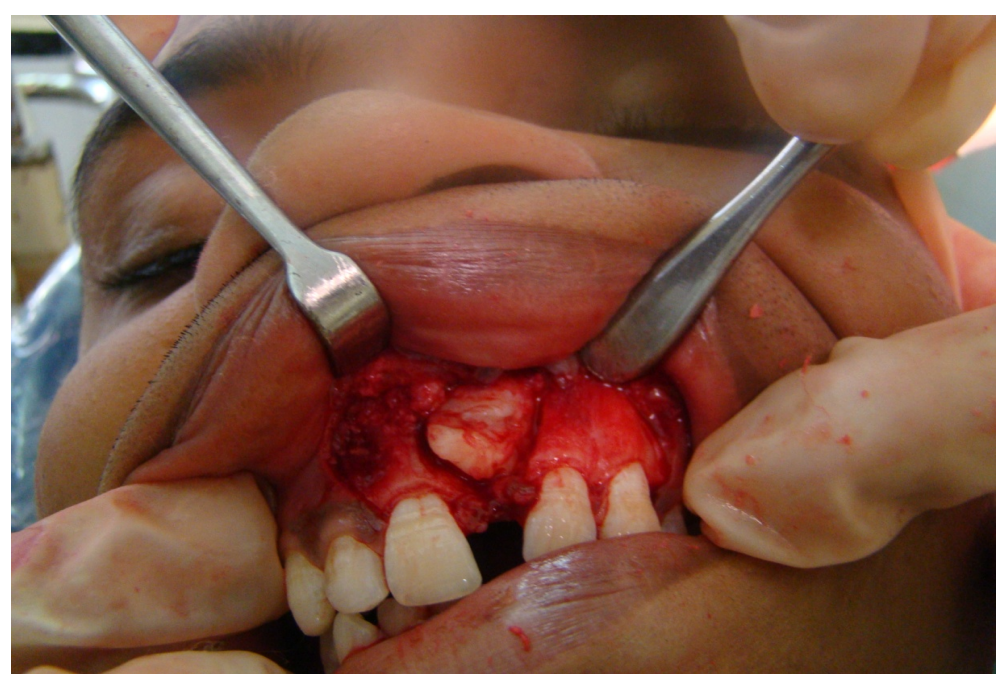

Figure 12. Showing intra operative exposure of central incisor.

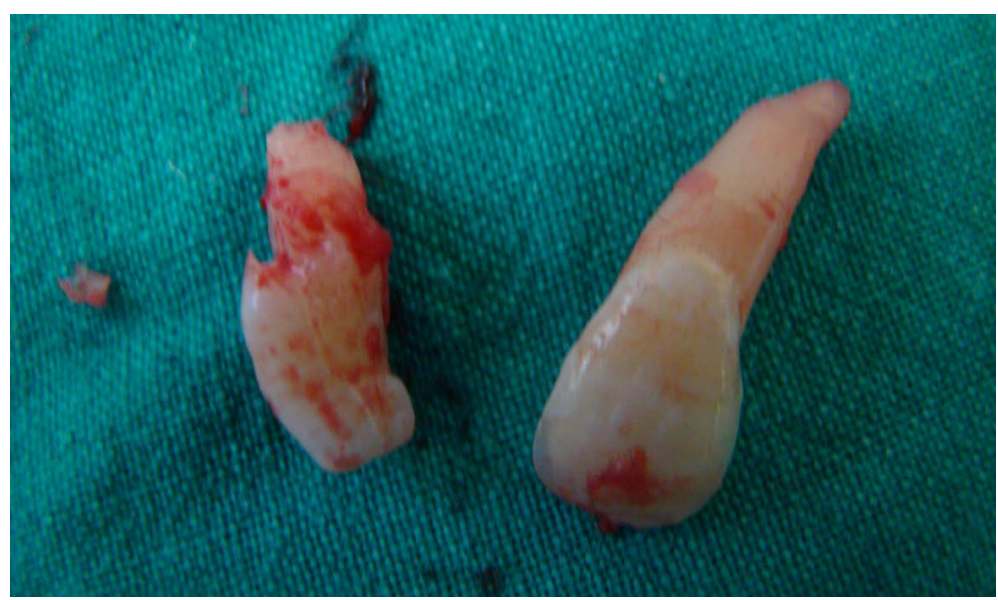

Figure 13. Maxillary central incisor \& odontoma.

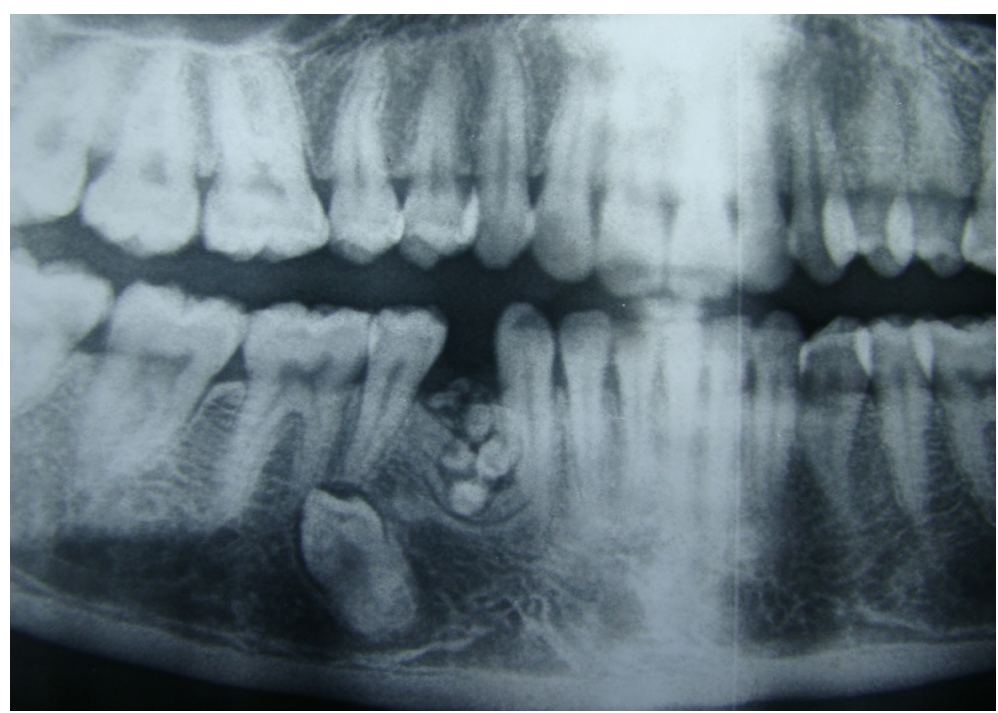

Figure 14. OPG showing odontoma (multiple teeth like structures) \& impacted tooth. 


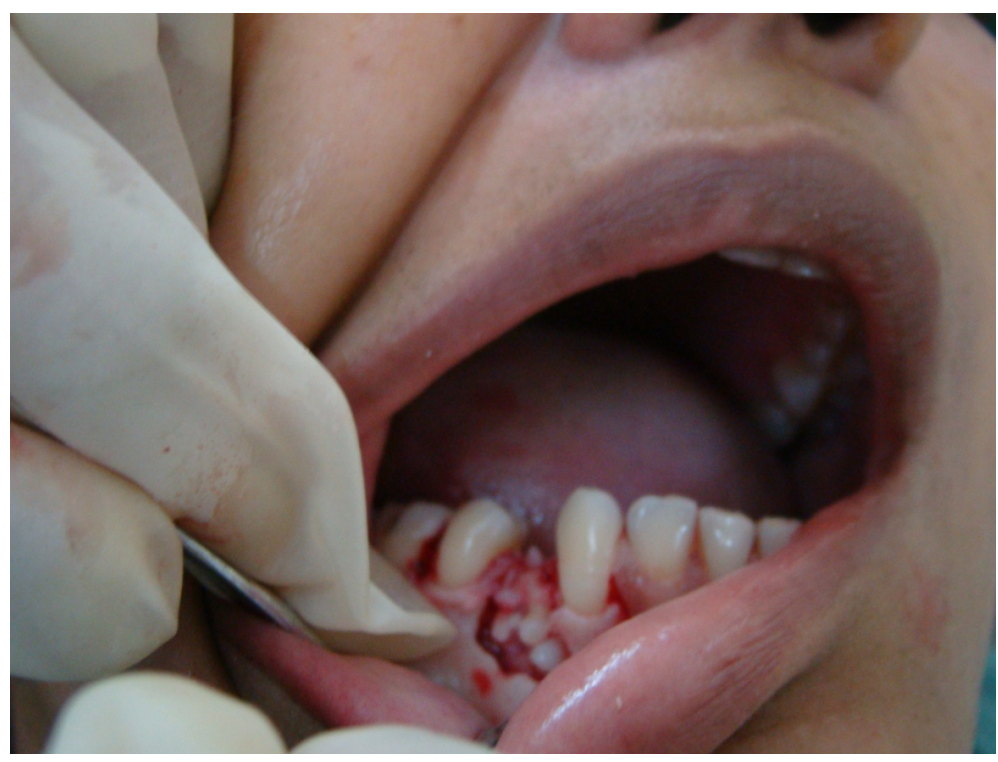

Figure 15. Intraoperative photo showing odontoma.

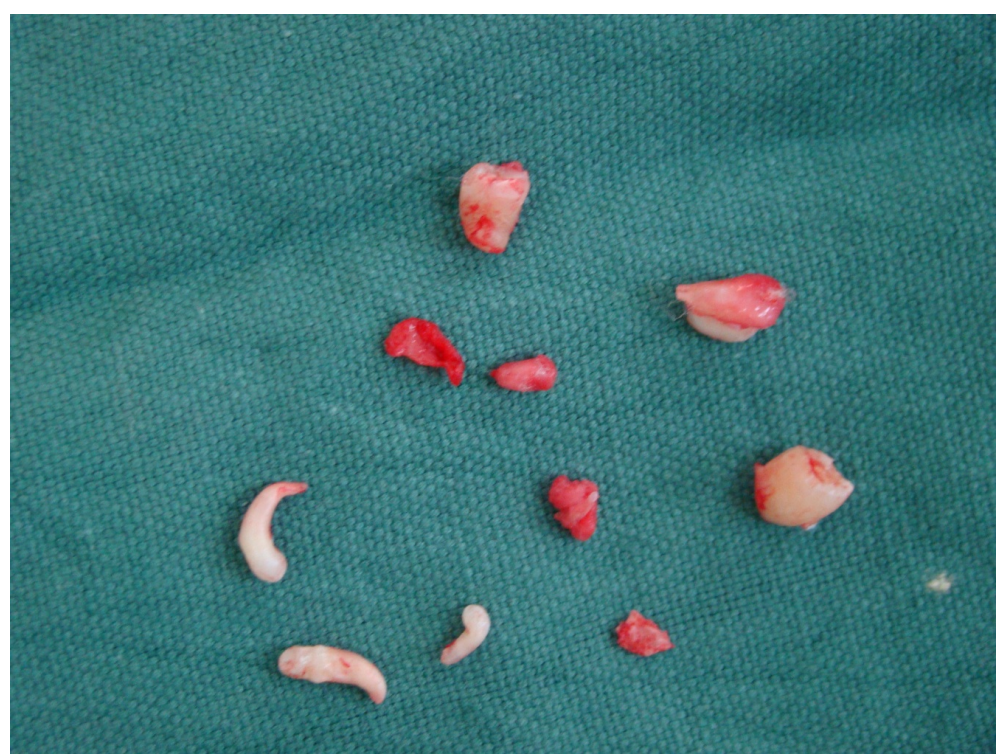

Figure 16. Multiple teeth like structures.

\section{Discussion}

The term odontoma was coined by Paul Broca in 1867. Odontomas are by definition hamartomas of odontogenic origin \& are usually considered developmental anomalies [2]. Odontomas are the most common odontogenic developmental anomalies found in the jaws [14].

With respect to location, odontomas were more frequently found in maxillary anterior region. In the studies of Regezi et al. [4] and Kaugars et al. [15] the most common site affected was the anterior region (incisors and canines) of the maxilla, followed by the anterior region of the mandible. Kaugars et al. [15] reported that the incidence of odontomas in the molar region gradually increases with each successive decade of life. Complex odontomas tend to occur more common in posterior mandible 44\%. [3] contrary to our three odontomas were present in mandibular posterior region \& only one compound odontomas was maxillary anterior region.

A classification given by $\mathrm{H}$ M Worth in 1937 is as follows [6].

- Epithelial odontomas arising from dental epithelium: Dental cyst, dentigerous cyst, multilocular cyst (ada- 
mantinoma).

- Composite odontmomes arising from the dental epithelium and dental mesoblastic tissues: Complex, compoundgeminated and dilated.

In 1946, Thoma and Goldman gave a classification which is as follows.

- Geminated composite odontomas: Two or more, more or less well-developed teeth fused together.

- Compound composite odontomas: Made up of more or less rudimentary teeth.

- Complex composite odontomas: Calcified structure bearing no great resemblance to the normal anatomical arrangement of dental tissues.

- Dilated odontomas: The crown or root part of tooth shows marked enlargement.

- Cystic odontomas: An odontoma that is normally encapsulated by fibrous connective tissue in a cyst or in the wall of a cyst.

According to World Health Organization (WHO) classification, odontomas can be divided into three groups.

- Complex odontoma: When the calcified dental tissues are simply arranged in an irregular mass bearing no morphologic similarity to rudimentary teeth.

- Compound odontoma: Composed of all odontogenic tissues in an orderly pattern, which result in many teeth-like structures, but without morphologic resemblance to normal teeth.

- Ameloblastic fibro-odontoma: Consists of varying amounts of calcified dental tissue and dental papilla-like tissue, the later component resembling anameloblastic fibroma. The ameloblasticfibro odontoma is considered as an immature precursor of complex odontoma.

There is no gender predilection and odontomas but considerable controversy exists over gender distribution. Some studies have shown odontomas more common in females than in males [16] [17], others consider these lesions to be similarly distributed both genders [18] [19]. Iatrous et al. \& Yadav et al. [20] [21] have shown male prediction similar to our study where male female ratio is 3:1.

Several case series have shown that the majority of odontomas were diagnosed in first two decades of life [18], similar to our study three patients were diagnosed in 2nd \& 3rd decade, they may be discovered at any age but less than $10 \%$ are found in over 40 years of age similar to our one case who is 45 years old.

Some studies have reported a correlation between patient age \& type of odontoma involved compound lesion being more frequent in younger patients [22], which is in support of our study. Compound odontoma was seen intwo patients between 18 - 24 years of age.

Several case series have documented that the majority of all odontomas occurred more frequently on the right side of jaw than on the left [1], in support of our study two patients showed odontomas on right side, one on left side posterior mandible \& one in anterior region.

Large odontomas are associated with local disturbances such as the eruption delay of permanent teeth and the development of cystic lesions as dentigerouscysts [3]. Rarely odontomas may form peripheral or soft tissue lesions in which they arise outside alveolar bone and may exfoliate or erupt [23]. The first case of an erupted odontoma was described in 1980 by Rumel et al. [24].

To the best of our knowledge only 20 cases have been documented in the literature. Of the 20 reported cases of erupted odontoma, 9 corresponded to compound and 11 to complex odontoma. This situation can cause pain, inflammation of the adjacent soft tissues, or infection associated with suppuration [25]. The mechanism of odontoma eruption appears to be different from tooth eruption because of the lack of periodontal ligament and root in odontoma. Therefore the force required to move the odontoma is not linked to the contractilitity of the fibroblasts, as in the case for teeth. Although there is no root formation in odontoma, its increasing size may lead to the sequestration of the overlying bone and, hence occlusal movement or eruption. An increase in the size of the odontoma over time produces a force sufficient to cause bone resorption [18]. Similar to our two cases which were erupted in oral cavity in posterior region\& showed signs of inflammation \& pain.

Although odontomas are commonly asymptomatic, clinical indicators may include retention of deciduous teeth, non eruption of permanent teeth, pain, expansion of cortical bone \& tooth displacement [2]. In the present series both pain \& absence of teeth was noted. Presence of pain can be probably due to secondary infection (as the odontoma were very close to alveolar mucosa) which can occur because of replacement of bone by large amount of avascular hard tissue [25].

Un erupted teeth are more common with compound than complex odontoma. [24] in contrast to present series where we found both complex \& compound odontomas were associated with un erupted teeth.

Discovery often occur due to radiographic investigation for the cause of non erupted permanent or retained 
deciduous teeth [18] [26] similar to our study impacted teeth are present in three out of four cases.

The etiology of the odontomas is unknown but the genetic factors and environmental causes such as trauma and infection have been proposed [13]. Lopez Areal has concluded in his study that if injury occurs early in childhood, it is more likely to form odontomas [27]. Hitchin [28] suggested that odontomas are inherited or due to a mutagene or interference possibly postnatal with the genetic control of tooth development.

Treatment included complete enucleation of odontomas intra orally small \& large \& all patients showed uneventful healing in contrast to Vibhasingh et al. [29] who have removed a large odontoma by submandibular approach.

\section{Conclusion}

Odontomas can be one of the causes for orofacial pain and swelling. There is the high association between odontomas and permanent teeth impaction as presented in our three cases. There is always danger of bone destruction leading to pathologic fractures if the lesion is left untreated. Intra oral approach is better than extra oral approach for removal of small/large odontomas thereby preventing the unnecessary scarring in young individuals for cosmetic reasons. Hence author recommends intra oral approach for removal of small as well as large odontomas.

\section{References}

[1] Shafer, W.G., Hine, M.K. and Levy, B.M. (1993) A Text Book of Oral Pathology. 4th Edition, WB Saunders \& Co., Philadelphia, 308-312.

[2] Vengal, M., Arora, H., Osh, S. and Pai, K.M. (2007) Large Erupting Complex Odontoma—A Case Report. Journal of the Canadian Dental Association, 73, 169-172.

[3] Shafer, W.G., Hine, M.K. and Levy, B.M. (1983) Text Book of Oral Pathology. 4th Edition, West Washington Square, WB Saunders Company, Philadelphia.

[4] Regezi, J.A. and Sciubba, J.J. Oral Pathology Clinical Pathologic Correlation. 3rd Edition, WB Saunders, Philadelphia, 350-352.

[5] Cawson, R.A., Binnie, W.H. and Eveson, J.W. (1993) Color Atlas of Oral Disease. Clinical and Pathological Correlations. Mosby-Wolfe, Hong Kong, 6-19.

[6] Owens, B.M., Schuman, N.J., Mincer, H.H., Turner, J.E. and Oliver, F.M. (1997) Dental Odontomas: A Retrospective Study of 104 Cases. Journal of Clinical Pediatric Dentistry, 21, 261-264.

[7] Katz, R.W. (1989) An Analysis of Compound and Complex Odontomas. ASDC Journal of Dentistry for Children, 56, 445-449.

[8] Bengston, A.L., Bengston, N.G. and Benassi, L.R.D.C. (1993) Odontomas em pacientes pediátricos. Revista de Odontopediatria, 2, 25-33.

[9] Philipsen, H.P., Reichart, P.A. and Praetorius, F. (1997) Mixed Odontogenic Tumours and Odontomas. Considerations on Interrelationship. Review of the Literature and Presentation of 134 New Cases of Odontomas. Oral Oncology, 33, 86-99. http://dx.doi.org/10.1016/S0964-1955(96)00067-X

[10] Daley, T.D., Wysoki, G.P. and Pringle, G.A. (1994) Relative Incidence of Odontogenic Tumors and Oral Jaw Cyst in a Canadian Population. Oral Surgery, Oral Medicine, Oral Pathology, Oral Radiology, and Endodontology, 77, 276280. http://dx.doi.org/10.1016/0030-4220(94)90299-2

[11] Ibsen, O.A. and Phelan, J.A. (2002) Oral Pathology for the Dental Hygienist. 3rd Edition, WB Saunders Co., Philadelphia, 626-632.

[12] Yıldırım-Öz, G., Tosun, G., Kızıloğlu, D., Durmuş, E. and Şener, Y. (2007) An Unusual Association of Odontomas with Primary Teeth. European Journal of Dentistry, 1, 45-49.

[13] Singh, S., Singh, M., Singh, I. and Khandelwal, D. (2005) Compound Composite Odontoma Associated with an Unerupted Deciduous Incisor-A Rarity. Journal of the Indian Society of Pedodontics and Preventive Dentistry, 23, 146150. http://dx.doi.org/10.4103/0970-4388.16889

[14] Mupparapu, M., Singer, S.R. and Rinaggio, J. (2004) Complex Odontoma of Unusual Size Involving the Maxillary Sinus: Report of a Case and Review of CT and Histopathologic Features. Quintessence International, 35, 641-644.

[15] Kaugars, G.E., Miller, M.E. and Abbey, L.M. (1989) Odontomas. Oral Surgery, Oral Medicine, Oral Pathology, Oral Radiology, and Endodontology, 67, 172-176. http://dx.doi.org/10.1016/0030-4220(89)90324-1

[16] Garcia-Consuegra, L., Junquera, L.M., Albertos, J.M. and Odontomas, R.O. (2000) A Clinical-Histological and Retro- 
spective Epidemiological Study of 46 Cases. Medicina Oral, 5, 367-372.

[17] Hisatomi, M., Asaumi, J., Konouchi, H., Honda, Y., Wakasa, T. and Kishi, K. (2002) A Case of Complex Odontoma Associated with an Impacted Lower Deciduous Second Molar and Analysis of the 107 Odontomas. Oral Diseases, 8, 100-105. http://dx.doi.org/10.1034/j.1601-0825.2002.1c778.x

[18] Nelson, B.L. and Thompson, L.D. (2010) Compound Odontoma. Head and Neck Pathology, 4, 290-291. http://dx.doi.org/10.1007/s12105-010-0186-2

[19] Serra-Serra, G., Berini-Aytés, L. and Gay-Escoda, C. (2009) Erupted Odontomas: A Report of Three Cases and Review of the Literature. Medicina Oral Patologia Oral y Cirugia Bucal, 14, E299-E303.

[20] Iatrous, I., Vardas, E., Theologie-Lygidakis, N. and Leventis, M. (2010) A Retrospective Analysis of the Characteristics, Treatment and Follow-Up of 26 Odontomas in Greek Children. Journal of Oral Science, 52, 439-447. http://dx.doi.org/10.2334/josnusd.52.439

[21] Yadav, M., Godge, P., Meghana, S. and Kulkarni, S. (2012) Compound Odontoma. Contemporary Clinical Dentistry, 3, S13-S15. http://dx.doi.org/10.4103/0976-237X.95095

[22] An, S., An, C. and Choi, K. (2012) Odontoma: A Retrospective Study of 73 Cases. Imaging Science in Dentistry, 42, 77-81. http://dx.doi.org/10.5624/isd.2012.42.2.77

[23] Chandra, S., et al. (2010) Compound Composite Odontoma Erupting into the Oral Cavity. Contemporary Clinical Dentistry, 1, 123-126. http://dx.doi.org/10.4103/0976-237X.68591

[24] Rumel, A., de Fritas, A., Birman, E., Tannous, L., Chacon, P. and Borker, S. (1980) Erupted Complex Odontoma: Report of a Case. Dentomaxillofacial Radiology, 9, 5-9.

[25] Nisha, D., Rishabh, K., Ashwarya, T., Sukriti, M. and Gupta, S.D. (2011) An Usual Case of Erupted Composite Complex Odontoma. Journal of Dental Sciences \& Research, 2, 1-5.

[26] Tuzum, M.S. (1990) Orofacial Pain Associated with an Infected Complex Odontoma. Case Report. Australian Dental Journal, 35, 352-354. http://dx.doi.org/10.1111/j.1834-7819.1990.tb00784.x

[27] Sood, P.B., et al. (2010) Multiple Supernumerary Tooth and Odontoma in the Maxilla: A Case Report. Contemporary Clinical Dentistry, 1, 45-46. http://dx.doi.org/10.4103/0976-237X.62528

[28] Shekar, S.E., et al. (2009) Erupted Compound Odontoma. Journal of Oral and Maxillofacial Pathology, 13, 47-50. http://dx.doi.org/10.4103/0973-029X.48758

[29] Singh, V., Dhasmana, S., Mohammad, S. and Singh, N. (2010) The Odontomas: Report of Five Cases. National Journal of Maxillofacial Surgery, 1, 157. http://dx.doi.org/10.4103/0975-5950.79220 
Scientific Research Publishing (SCIRP) is one of the largest Open Access journal publishers. It is currently publishing more than 200 open access, online, peer-reviewed journals covering a wide range of academic disciplines. SCIRP serves the worldwide academic communities and contributes to the progress and application of science with its publication.

Other selected journals from SCIRP are listed as below. Submit your manuscript to us via either submit@scirp.org or Online Submission Portal.
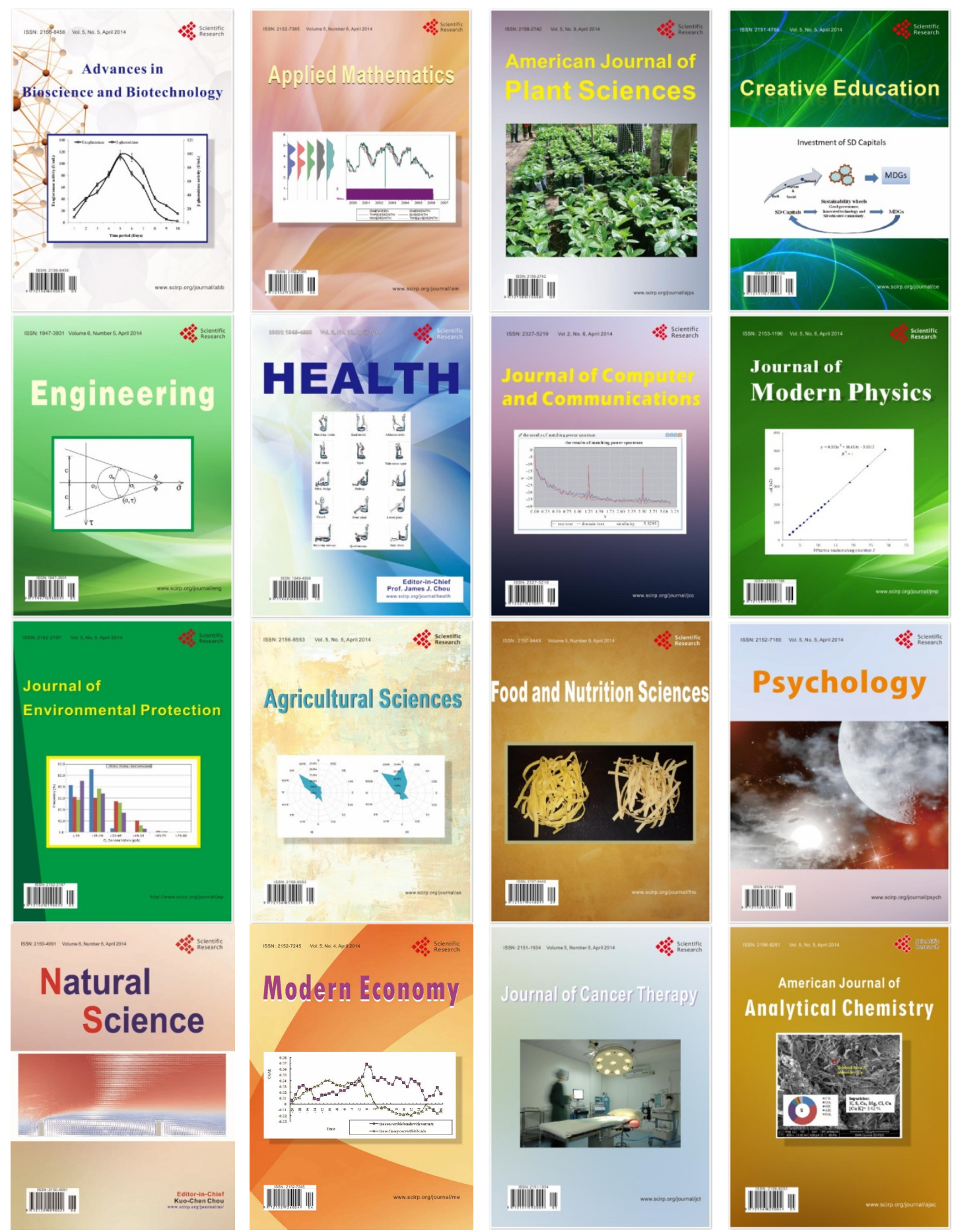